\title{
Study on Microwave-Absorbing Behavior of Multi-Walled CNTs
}

\author{
Xiaolai Liu (Corresponding author) \\ College of Science \\ Beijing University of Chemical Technology, Beijing 100029, China \\ E-mail: 11ltyx657@163.com
}

\begin{abstract}
To increase the complex permeability and widen the frequency band of carbon nanotubes (CNTs) are the most essential ways for promoting the microwave-absorbing property of microwave-absorbing coatings on multi-wall CNTs. Therefore, we have investigated the relations between the microwave-absorbing behavior and the complex permeability of CNTs, analyzed the electromagnetic parameters of CNTs/paraffin composites of different concentrations under different frequencies, and figured out the external conditions to obtain the optimum wave-absorbing behavior, which is particularly helpful for the further development of new material.
\end{abstract}

Keywords: CNTs, Complex permeability, Microwave-absorbing materials

\section{Introduction}

With the development of electronic technology, electromagnetic radiation has become a new pollution source, which not only causes interference in electronic circuits, but also has adverse effects on people's health (Planeix $\mathrm{J} \mathrm{M}$ et al, 2001, p. 1447). On the other hand, with the development of electromagnetic wave detection technology, the stealth of military target plays an important role in improving the overall defense and attack ability of weapon system. At present, electromagnetic wave-absorbing material (EAM) is the most efficient way to reduce the electromagnetic wave pollution and realize the stealth of target. EAM absorbs the energy in electromagnetic waves as magnetic loss, and converts that energy, in the end, to heat. EAM currently has been widely used not only for the military purposes, such as stealth and anti-stealth, countermeasures and anti-countermeasures, but for such things as safety protection against radiation, microwave darkroom, EMI suppression of communication system and navigational aid, secret communication, improvement of machine's overall performance, increase of signal/noise ratio, electromagnetic compatibility (EMC), coaxial absorption unit, and etc (Cheng $\mathrm{H} \mathrm{M}$ et al, 2001, p. 1317).

EMA is desired to have a strong broadband absorption, low density, thin thickness and electrical stability. However, the traditional EMA can hardly meet the above comprehensive requirements. CNTs, first reported in 1991 by S. Iijima, a Japan specialist in electron microscopy (EM), have been widely studied in recent years due to their unique features. The experiment results have indicated that EMA made out of CNTs has many advantages, such as strong broadband absorption, low density, stable performances, and etc., can perfectly meet the practical application requirements, and is a new wave-absorbing material.

CNTs are allotropes of carbon with a cylindrical nanostructure. These cylindrical carbon molecules have novel properties that make them potentially useful in many applications in nanotechnology, optics, electronics, magnetics and other fields of materials science. The diameter of CNTs $(1 \sim 100 \mathrm{~nm})$ is much shorter than the wavelength of IR and radar radiation, therefore, nanomaterials have much stronger IR-absorption or microwave-absorption than the traditional materials have.

In recent years, the studies on wave-absorption performances of CNTs mainly include CNTs films, activated CNTs EAM, magnetic alloy/CNTs hybrids, and polymer/CNTs hybrids. There mainly have been three issues addressed in these studies.

(1) CNTs thin film, a combination of the nanotechnology and the thin film technology, exhibits a combined feature of the thin films and the nanomaterials, and becomes one of the hottest research topics in materials science.

(2) CNTs have been constructed with a very large length-to-diameter ratio, and frequently referred to as "one-dimensional". Their one-dimensional hollow structures, exactly as capillary tubes, can draw some elements into tubes, and form one-dimensional quantum lines of special performances. Although CNTs show no magnetic features, by coating magnetic metal on the tubes or filling magnetic iron into the tubes, they can form $\mathrm{CNTs} /$ magnetic chains hybrids, which exhibit both magnetic properties and electro conductivities, and can be used to make microwave-absorbing materials of low density and strong absorption through the loss of electromagnetic energy. Compared with no-filling materials, metal-contained multi-walled CNTs exhibit much 
more reflection loss and much larger bandwidth. Metal coating has obvious effects on the dielectric constant and magnetic properties of CNTs, which will, in turn, affect the microwave absorption of the end product (John W et al, 2003).

(3) Conductive network can be achieved by introducing polymer into CNTs. Therefore, polymer/CNTs hybrids, as new structural and functional materials, can be used to make wave-absorbing materials of broadband absorption (Roberts J A et al, 2004, p. 4352-4356).

The wave-absorption of materials can be determined from electromagnetic parameters, such as permeability $\left(\mu_{\mathrm{r}}\right)$ and dielectric constant $\left(\varepsilon_{\mathrm{r}}\right)$. To increase the complex permeability and widen the frequency band of CNTs are the most essential ways for promoting the wave-absorbing properties of the coated wave-absorbing CNTs. Therefore, it is important to study the directional effective permeability of CNTs. This paper has measured and collected the permeability of single-walled, as well as multi-walled CNTs/paraffin composites of different concentrations in the range of $2 \sim 18 \mathrm{GHz}$, constructed the graphs by the Origin software under different conditions, and analyzed the effects of concentration of composite and frequency on the permeability of CNTs.

\section{Experiment and measurement}

\subsection{Measurement of sample}

Take untreated multi-walled CNTs as the target samples, and measure the dielectric constant and the complex permeability of multi-walled CNTs/paraffin composites of different concentrations in the range of 2 18 GHz. Figure 1 exhibits a SEM image of multi-walled CNTs.

\subsection{Measurement of electromagnetic parameters of CNTs/paraffin composites}

Electromagnetic parameters of CNTs/paraffin composites in the range of $2 \sim 18 \mathrm{GHz}$ are determined based on the coaxial line method. A range of $10 \mathrm{MHz}$ to $26.5 \mathrm{GHz}$ signal generated by HP83630L (Hewlett Packard 83860L-Seriers Swept-CW Generator) passes through the target sample, and is then detected by HP8722ES Network Analyzer.

Pure CNTs are too active to be compacted. As a result, it is hardly to obtain the desired shapes and determine their electromagnetic parameters directly. Furthermore, the melting point of paraffin is relatively low (about $60{ }^{\circ} \mathrm{C}$ ), the real and imaginary parts of the complex dielectric constant of paraffin in the range of $2 \sim 18 \mathrm{GHz}$ are 2.23 2.27 and 0 0.01, respectively, and its dielectric loss is close to zero. Therefore, paraffin is commonly used as a binder for determination of the electromagnetic parameters of CNTs (Bower C et al, 2002, p. 3820; Zhao D L et al, 2001, p. 2471). Disperse CNTs uniformly in the fused paraffin, and cast this liquid into a ring-shaped standard copper flange to obtain a ring-shaped target sample of $3.0 \mathrm{~mm}$ inner diameter, $7.0 \mathrm{~mm}$ outer diameter and $2.0 \mathrm{~mm}$ thickness. The real and imaginary parts of the complex dielectric constant and complex permeability of CNTs can be directly detected by accessing the above sample-filled flange into the test system.

\section{Results and Discussions}

Determine the real and imaginary parts of electromagnetic parameters of multi-walled CNTs/paraffin composites of five different concentrations in the range of $2 \sim 18 \mathrm{GHz}$, then graph and analyze the obtained data by an excellent software: Origin. The test results of both single-walled and multi-walled CNTs/paraffin composites are compared for further study on the selection of wave-absorbing materials.

\subsection{Relation between the real part of permeability of multi-walled CNTs and frequency}

Figure 2 exhibits the relation between the real part of permeability of multi-walled CNTs and frequency.

In figure $2, \mu_{1}^{\prime} \sim \mu^{\prime}{ }_{5}$ represent the real parts of permeabilities of multi-walled CNTs with the concentrations of $1 \%$, $5 \%, 10 \%, 15 \%$ and $20 \%$, respectively. From it, we can see that the real part of permeability is close to 1 and almost keeps constant despite the increase of frequency when the concentration of CNTs is relatively low, such as $1 \%$ and $5 \%$, respectively; changes a little in the range from 0.9 to 1.05 with the increase of frequency when the concentration is up to $10 \%$; and changes obviously with the increase of frequency when the concentration is relatively high, such as up to $15 \%$ and $20 \%$, respectively. Take the curve of $20 \%$ for example, at low frequency (from $2 \mathrm{GHz}$ to $8 \mathrm{GHz}$ ), its real part is 0.89 at the frequency of $2 \mathrm{GHz}$, then sharply decreases with the rise of frequency, and is down to 0.53 at the frequency of $8 \mathrm{GHz}$; whereas at high frequency (higher than $8 \mathrm{GHz}$ ), its real part almost keeps constant again, and changes a little with the rise of frequency. These results indicate that $\mu$ ' is related to the concentration in a given frequency range: at a low concentration (lower than $10 \%$ ), $\mu$ ' almost keeps constant despite the increase of frequency; whereas at a high concentration (such as $15 \%$ and $20 \%$ ), $\mu$ ' sharply decreases with the rise of frequency in the range from $2 \mathrm{GHz}$ to $8 \mathrm{GHz}$, and changes a little with the rise of frequency in the range from $12 \mathrm{GHz}$ to $18 \mathrm{GHz}$, or even higher. 


\subsection{Relation between the imaginary part of permeability of multi-walled CNTs and frequency}

Figure 3 exhibits the relation between the imaginary part of permeability of multi-walled CNTs and frequency.

In figure $3, \mu "{ }_{1} \sim \mu{ }_{5}$ represent the imaginary parts of permeabilities of multi-walled CNTs with the concentrations of $1 \%, 5 \%, 10 \%, 15 \%$ and $20 \%$, respectively. From it, we can see that $\mu$ " is in the range of $-0.15 \sim 0.1$. At a low concentration such as $10 \%$ or lower, $\mu$ " is negative, and almost keeps constant despite the rise of frequency; whereas at a high concentration, $\mu$ " changes a little with the rise of frequency, and alternates between the negative and positive; when the concentration is up to $20 \%, \mu$ " changes obviously in the positive range at low frequency, and reaches the peak of 0.27 at $5.2 \mathrm{GHz}$. These results indicate that $\mu$ " is related to the concentration in a low frequency range: $\mu$ " changes obviously with the rise of the concentration; whereas in a high frequency range, $\mu$ " almost keeps constant despite the change of frequency. It's probably because the magnetic field change has an obvious effect on the magnetic dipole array, but the magnetic dipole array lags behind the magnetic field change in the high frequency range. It is also observed that $\mu$ "of multi-walled CNTs is high than that of single-walled CNTs, and exhibits a rhythmic change. Therefore, the study on the imaginary part of permeability is very valuable for the development of high-performance wave-absorbing materials.

\subsection{Relation between the loss tangent of permeability of multi-walled CNTs and frequency}

Figure 4 exhibits the relation between the loss tangent of permeability of multi-walled CNTs and frequency.

In figure $4, \tan \delta_{1} \sim \tan \delta_{5}$ represent the loss tangent of permeabilities of multi-walled CNTs with the concentrations of $1 \%, 5 \%, 10 \%, 15 \%$ and $20 \%$, respectively. Usually, the larger the loss tangent $(\tan \delta)$ is, the better the wave-absorbing materials are. That is, as to $\mu$ ", the higher the better; however, as to $\mu^{\prime}$, the lower the better.

From figure 4, we can also see that in a low concentration range, $\tan \delta$ almost keeps constant despite the rise of frequency; whereas in a high concentration range, $\tan \delta$ fluctuates with the rise of frequency, and changes much more obviously when the concentration increases. Therefore, the peak usually appears in the high concentration range.

\section{Conclusion}

(1) The frequency spectra of the real part, imaginary part, as well as loss tangent of permeability of multi-walled CNTs exhibit the similar patterns: in a low concentration range, they almost keep constant despite the rise of frequency; whereas in a high concentration range, they fluctuate with the rise of frequency, and change much more obviously when the concentration is higher. Therefore, their peaks usually appear in the low-frequency high-concentration range.

(2) In a high frequency range, the real part, imaginary part, as well as loss tangent of permeability of multi-walled CNTs of the same concentration almost keep constant despite the change of frequency. It's probably because the magnetic field change has an obvious effect on the magnetic dipole array, but the magnetic dipole array lags behind the magnetic field change in the high frequency range.

(3) The loss tangent of permeability of multi-walled CNTs is similar to that of single-walled CNTs when the concentration is low (Mouchon E et al, 1996, p. 323; Li J D et al, 1999, p. 721). However, when the concentration is high, especially in a low frequency range, the loss tangent of multi-walled CNTs is much higher than that of single-walled CNTs (the peak of the former is up to 0.39 , whereas that of the latter is only 0.03 ), and exhibits a rhythmic change. It demonstrates that as far as the loss tangent is concerned, the wave-absorption of multi-walled CNTs is better than that of single-walled CNTs.

\section{References}

A K Jonscher. (1983). Dielectric Relaxation in Solids. London: Chelsea Dielectric Press. p. 138.

C Bower, W Zhu, D Shalom, D Lopez, P L Gammel \& S Jin. (2002). Appl. Phys. Lett, 80, 3820.

Cheng H M, Yand Q H \& Liu C. (2001). Carbon, 294, 1317.

E Mouchon \& Ph Colomban. (1996). J. Mater. Sci., 31, 323.

J A Roberts, T lmholt \& Z Ye. (2004). Journal of Applied Physics, 95, 4352-4356.

John W Schultz \& Rick L Moore. (2003). Effective medium calculations of the electromagnetic behavior of single walled carbon nanotube composites. Materials Research Society Symposium-Proceedings, 739.

K G Ong, K Zheng \& C Grimes. (2002). IEEE Sensors J, 2, 82.

Li, Jingde, Chen, Min, Fang, Chuandai, Li, Zhiqiang \& Lei, Deming. (1999). Dielectric spectroscopy investigation on polar phase transitions. Acta Physica Sinica, 48(4), 721-728. 
P Debye. (1945). Polar Molecules. New York: Dover. p. 257.

Planeix J M, Coustel N \& Coq B. (2001). J Am Carbon, 39, 1447.

Service R F. (1998). Science, 281, 940.

Zhao, Donglin, Zhou, Wancheng \& Wan, Wei. (2001). Microwave permittivity of nano Si/C/N composite powders. Acta Physica Sinica, 50(12), 2471-2476.

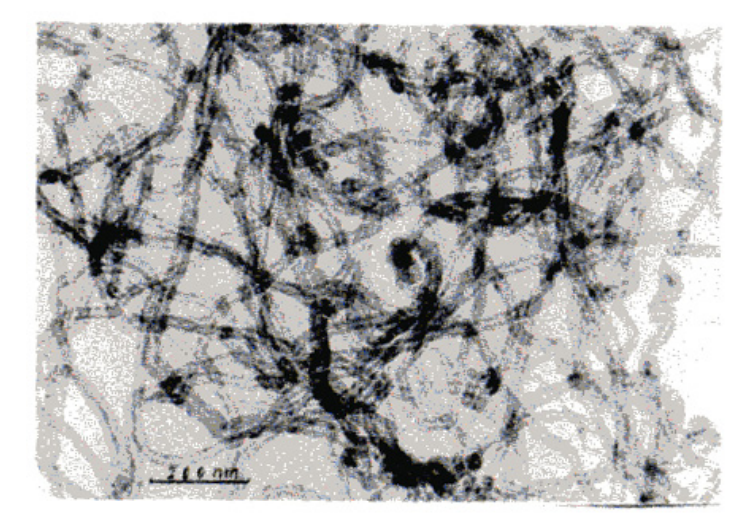

Figure 1. SEM image of multi-walled CNTs

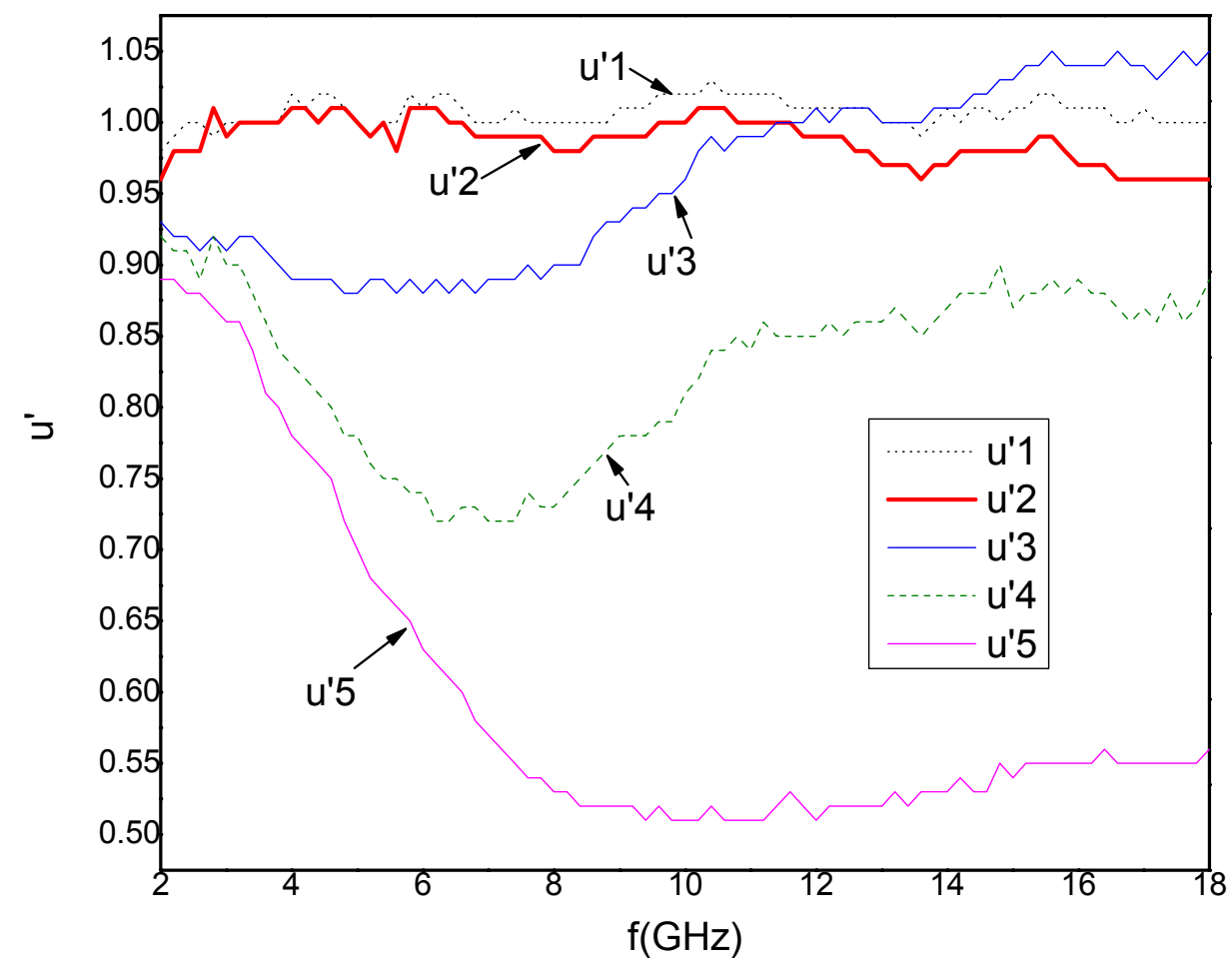

Figure 2. Relation between the real part of permeability of multi-walled CNTs and frequency 


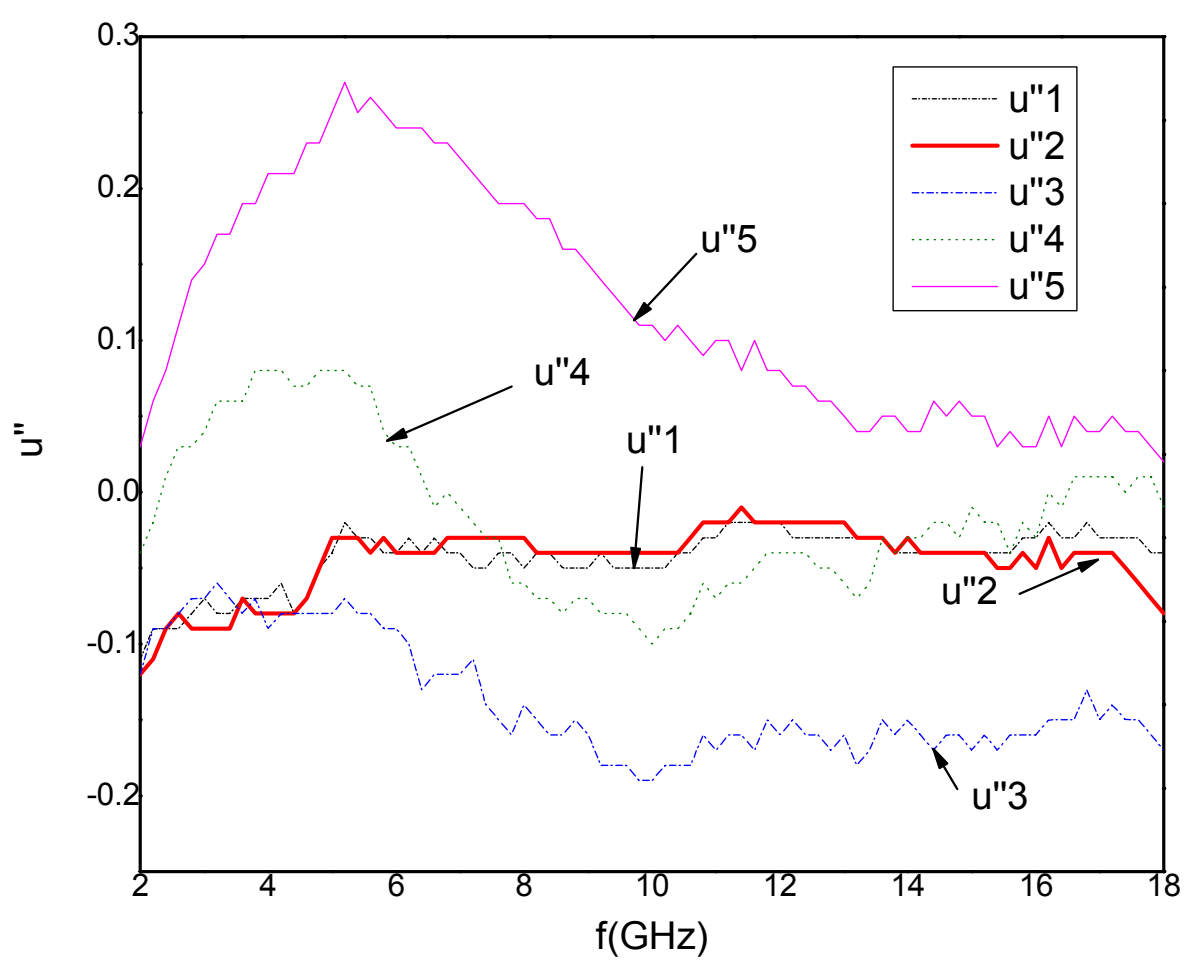

Figure 3. Relation between the imaginary part of permeability of multi-walled CNTs and frequency 


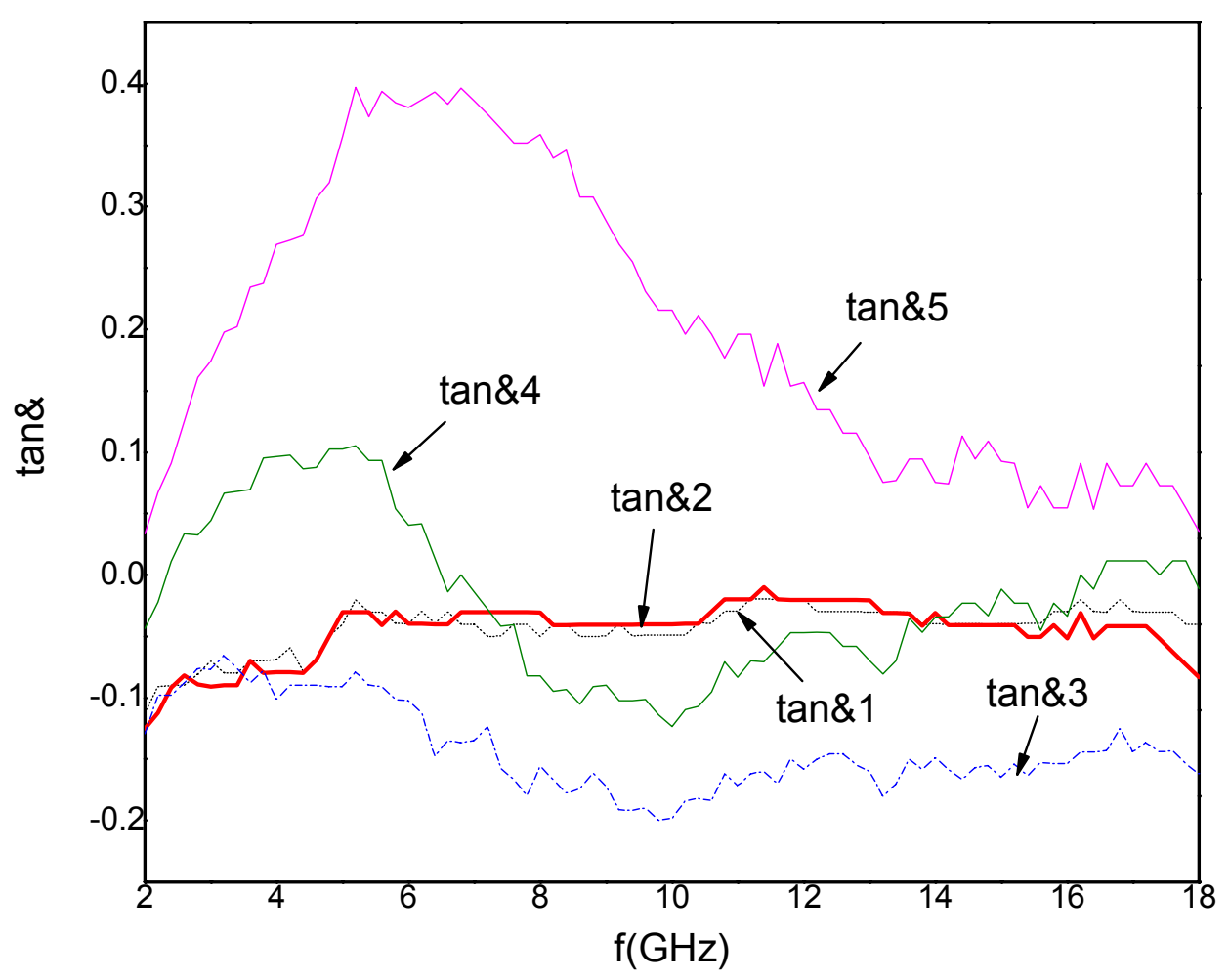

Figure 4. Relation between the loss tangent of permeability of multi-walled CNTs and frequency 